\title{
Assessment of Macular and Retinal Nerve Fiber Layer Changes after Uncomplicated Phacoemulsification Surgery in Diabetic Patients Using Optical Coherence Tomography
}

M.F.El-khiat, M.A.Tabl, and M.I.A.Ramadan

Ophthalmology, Dept., Faculty of Medicine, Benha Univ., Benha, Egypt

E-mail:Mohamed.abouelea@hotmail.com

\begin{abstract}
Background: Macular edema (ME) is a major cause of vision loss after cataract surgery in patients with diabetes. In this study we aimed to assess the macular and retinal nerve fiber layer changes after uncomplicated cataract surgery in the early postoperative period in diabetic and non-diabetic patients. Methods: This is a comparative cross-sectional study conducted on patients with cataract, included 30 patients divided into 2groups: Group 1 (Study group): Diabetes mellitus group (DM group) consists of 15 eyes with cataract (15 diabetic patients without diabetic retinopathy). Group 2 (Control group): non diabetic group consist of 15 eyes with cataract (15 nondiabetic patients). Preoperative and 30 days postoperative OCT measurements were compared. All patients were admitted for cataract surgery (phacoemulsification) and posterior chamber intraocular lens (IOL) in Ophthalmology Department Benha University Hospital. Results and conclusion: The mean $( \pm \mathrm{SD})$ age of diabetic group was $57.7( \pm 6.4)$. They were 6 males $(40 \%)$ and 9 females $(60 \%)$. While the mean $( \pm \mathrm{SD})$ age of non-diabetic group was $60.2 \pm(9.5)$. They were 9 males $(60 \%)$ and 6 females $(40 \%)$. Both groups were matched regarding age and gender ( $p>0.05$ for both). Our data indicated that, although in each group; there is a significant increase in MFT and peripapillary RNFL postoperatively. However, there is a significant statistical difference between both groups regarding MFT and peripapillary RNFL after 30 days postoperative. Also there is improvement in BCVA progressively postoperative in each group, but there is a statistical significant difference between both groups.
\end{abstract}

Keywords: Macular, Retinal, Phacoemulsification, Diabetic, Optical Coherence Tomography.

\section{Introduction}

Diabetic patients pose a particular challenge because of the tendency for early formation of cataract in them and propensity to develop macular edema after cataract surgery. Macular edema (ME) is a major cause of vision loss after cataract surgery in patients with diabetes [1].

Macular edema is a common cause of unfavorable visual outcome after cataract surgery. Clinically significant cystoid macular edema (CSME) has a reported incidence of $1 \%$ to $2 \%$ after cataract surgery. Diabetes has been associated with an increased incidence of postoperative macular edema. The incidence of macular edema on optical coherence tomography (OCT) was $22 \%$ in diabetic eyes undergoing cataract surgery. The macular edema after cataract surgery in diabetic patients could be caused by the cataract surgery or diabetes itself, but it might be hard to differentiate between these two causes [2].

Optical coherence tomography (OCT) has been established as a practical method for examining retinal architecture. OCT, with its noninvasive nature, has been proven to be an indispensable tool for diagnosing retinal pathologies, including cystoid macular edema (CME). Many studies have reported incidences of CME and macular thickness changes, determined by OCT following uneventful cataract surgeries [3].

Many previous studies have used the optical coherence tomography (OCT) and have reported that the retinal nerve fiber thickness measurements can be increased after uncomplicated cataract surgery because the lens opacity of cataract may affect the retinal nerve fiber thickness measurements [4].
These studies found that the increase in the retinal nerve fiber layer (RNFL) thickness measurement after phacoemulsification surgery is because of improvement in transmittance and reflectivity of the RNFL boundary after removal of opacified lens, rather than actual RNFL thickening after cataract surgery [5].

OCT has been shown to be highly reproducible in measuring macular thickness in normal individuals and diabetic patients. For detecting macular edema, OCT is superior to contact lens biomicroscopy and as effective as fundus fluorescein angiography (FFA). Since OCT can assess macular thickness quantitatively, it can detect subtle changes of macular thickness and is especially useful in mild cases [6].

Macular edema may be related to impairment of the blood retinal barrier and an increased susceptibility to surgical trauma in diabetic patients. Other factors that may contribute to the progression of diabetic retinopathy (DR) and possibly to an increased incidence of CME after phacoemulsification in diabetics may include chronic inflammatory mechanisms [7].

To differentiate the effect of cataract surgery from the natural course of the disease, a prospective study which showed that uncomplicated phacoemulsification cataract surgery does not accelerate progression of DR as smaller incision size and shorter surgical time in phacoemulsification decrease inflammation and may induce less breakdown of the blood ocular barrier. The ETDRS study, however, suggested a trend toward increased retinopathy progression and worsening VA 
in eyes undergoing cataract surgery compared to unoperated fellow eyes in people with diabetes [8].

Increase in mean foveal thickness (MFT) occurred after uncomplicated phacoemulsification in diabetic and nondiabetic eyes; the range of increased MFT was more in diabetic patients with higher initial MFT or higher grade of DR preoperatively [9].

In this study we aimed to assess the macular and retinal nerve fiber layer changes after uncomplicated cataract surgery in the early postoperative period in diabetic and non-diabetic patients.

\section{Patients and methods}

This is a comparative cross-sectional study conducted on patients with cataract attending outpatient clinic of Ophthalmology Department Benha University Hospital. This study included 30 patients divided into 2groups:

- Group 1 (Study group): Diabetes mellitus group (DM group) consists of 15 eyes with cataract (15 diabetic patients with type 2 Diabetes mellitus without diabetic retinopathy).

- Group 2 (Control group): non diabetic group consist of 15 eyes with cataract (15 nondiabetic patients).

All patients were admitted for cataract surgery (phacoemulsification) and posterior chamber intraocular lens (IOL) in Ophthalmology Department Benha University Hospital.

\subsection{Exclusion criteria were as follow:}

- Patient with dense cataract or vitreous hemorrhage interfering with measurement of central macular thickness

- Diabetic retinopathy

- Chronic uveitis

- Any posterior segment pathology that could affect retinal thickness

- Evidence of vitreomacular traction by OCT

- Patient with intraoperative or postoperative complication (posterior capsule rupture with or without vitreous loss or dropped nucleus or nuclear fragments or iris trauma or postoperative inflammation or corneal edema).

\subsection{All patients were subjected to thorough history} taking, and Ocular examination as:

- Best corrected visual acuity (BCVA) using the Snellen's Decimal distance visual acuity preoperative and then 1 month postoperative.

- Preoperative anterior segment examination with slit lamp biomicroscopy to assess cataract density.

- Preoperative measurement of intraocular pressure (IOP) with applanation tonometer.

- Preoperative fundus examination with slit lamp biomicroscopy with $+78 \mathrm{D}$ lens and indirect ophthalmoscopy to assess macular status and peripheral retina.

\subsection{Phacoemulsification}

- One hour before surgery, the pupil was dilated with tropicamide $1 \%$.

- Peribulbar anaesthesia was used in all cases.

- The technique of the surgery as follow, sterilization of the periocular skin with Povidone iodine $10 \%$ washing conjunctival sac with Povidone iodine 5\% for 3 minutes

- Clear corneal incision was made by angled $2.8 \mathrm{~mm}$ disposable metal keratome with 2 side ports 90 apart using MVR 20 gauge, the anterior chamber was filled with Hydroxypropyl Methylcellulose, then capsulorhexis was done using capsulorhexis forceps

- OVD was evacuated and hydrodissection and rotation of the nucleus was done.

- Phacoemulsification was done and parameters including phacol which was used for making the trench, phaco 2 used for quadrant removal and effective phaco time (EPT) was recorded.

- All cases were done using stop and chop technique.

- Cortical removal using bimanual I/A, a tangential stripping method was used in combination with gentle centripetal movements to allow cortical material to separate from the capsular bag.

- Implantation of a PCIOL and hydration of the main wound and side ports

- All surgeries had been done by same surgeon

\subsection{Post-operative follow up}

All patients used moxifloxacin $0.5 \%$ and prednisolone acetate $1 \%$ eye drops five times a day for 2 weeks and tapered in 2 weeks. All patients were followed up for 1 month

\subsection{Optical Coherence tomography (OCT)}

- Pupils was dilated for OCT examination in all cases with $1 \%$ tropicamide (Mydrapid)

- OCT was done preoperatively and then at 1 month postoperatively using Topcorn 3D OCT- 2000 FA optical coherence tomography.

- CMT (Central Macular Thickness) and Peripapillary RNFL (Retinal nerve fiber layer) was measured.

\subsection{Statistical analysis}

The collected data was revised, coded, tabulated and introduced to a PC using Statistical package for Social Science (IBM Corp. Released 2017. IBM SPSS Statistics for Windows, Version 25.0. Armonk, NY: IBM Corp.). Data were presented and suitable analysis was done according to the type of data obtained for each parameter. Descriptive statistics: Mean, Standard deviation $( \pm \pm \mathrm{SD})$ was used to describe parametric numerical data. Frequency and percentage of nonnumerical data. Analytical statistics: Student T Test was used to assess the statistical significance of the difference between two study group means. Paired sample $t$ test was used to assess changes in parameters 
over time. Correlation analysis: To assess the strength of association between two quantitative variables. The correlation coefficient defines the strength and direction of the linear relationship between two variables.

\section{Results}

The mean $( \pm \mathrm{SD})$ age of diabetic group was 57.7 $( \pm 6.4)$. They were 6 males $(40 \%)$ and 9 females $(60 \%)$. While the mean $( \pm \mathrm{SD})$ age of non-diabetic group was $60.2 \pm(9.5)$. They were 9 males $(60 \%)$ and 6 females $(40 \%)$. Both groups were matched regarding age and gender ( $p>0.05$ for both). Table (1)

No significant differences were found between diabetics and non-diabetics regarding preoperative BCVA (p1>0.05). Diabetics showed significantly lower postoperative BCVA when compared non diabetics $(\mathrm{p} 1<0.001)$. Regarding non diabetics, BCVA increased significantly after operation $(\mathrm{p} 2<0.001)$.
Regarding diabetics, BCVA increased significantly after operation (p2<0.001). fig. (1)

Diabetics showed significantly higher pre as well as postoperative CMT when compared non diabetics (p1=0.010, 0.001 respectively). Regarding non diabetics, CMT increased significantly after operation (p2<0.001). Regarding diabetics, CMT increased significantly after operation (p2<0.001). fig. (2)

No significant differences were found between diabetics and non-diabetics regarding preoperative as well as postoperative RNFL thickness (p1>0.05 for each). Regarding non diabetics, RNFL thickness increased significantly after operation (p2<0.001). Regarding diabetics, RNFL thickness increased significantly after operation (p2<0.001). fig. (3)

No significant correlations were found between postoperative BCVA with preoperative CMT and RNFL thickness among studied groups. Table (2)

Table (1) Comparison of age and gender between diabetics and control groups.

\begin{tabular}{llcccccc}
\hline & \multicolumn{2}{c}{ Non diabetics } & \multicolumn{2}{c}{ Diabetics } & \multicolumn{2}{c}{ P } & Significance \\
\hline N=15e (years) & mean \pm \pm SD & 60.2 & 9.5 & 57.7 & $6 . \varepsilon$ & 0.400 & NS \\
Male & $\mathbf{N}, \%$ & 9 & $60 \%$ & 6 & $40 \%$ & 0.273 & NS \\
Female & $\mathbf{N}, \%$ & 6 & $40 \%$ & 9 & $60 \%$ & & \\
\hline
\end{tabular}

$\mathrm{NS}=$ non-significant
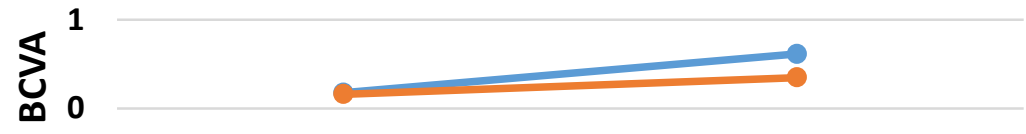

Preoperative

Postoperative

Non diabetics

Diabetics

Fig. (1) Pre and post-operative BCVA among diabetics and control groups.

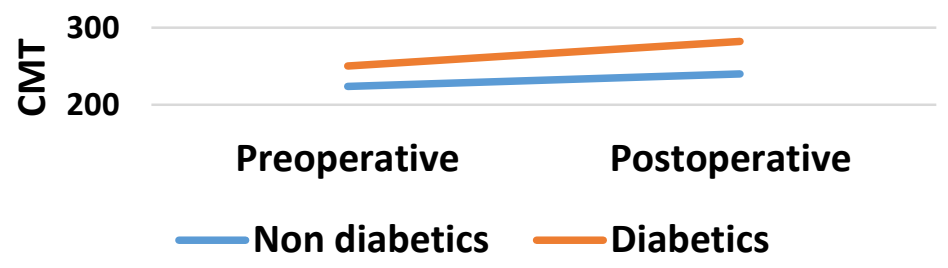

Fig. (2) Pre and post-operative CMT among diabetics and control groups.

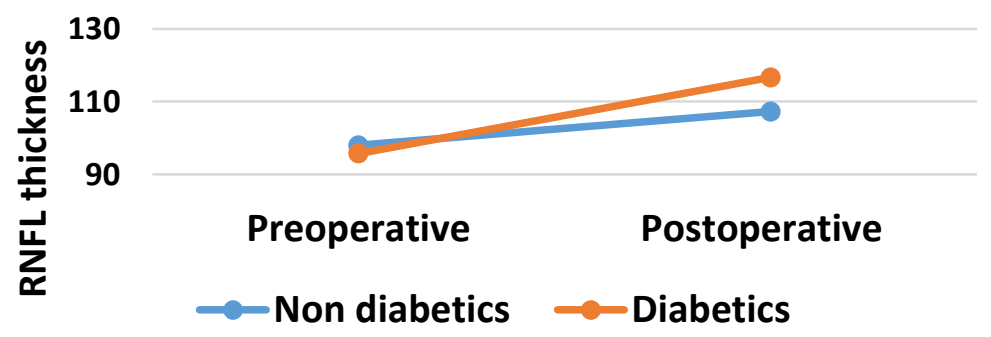

Fig. (3) Pre and post-operative RNFL thickness among diabetics and control groups. 
Table (2) Correlation of postoperative Best Corrected Visual Acuity with Central Macular Thickness and Retinal Nerve Fiber Layer thickness among studied groups.

\begin{tabular}{lcccc}
\hline & \multicolumn{3}{c}{ postoperative BCVA } \\
& Non diabetic group & \multicolumn{2}{c}{ Diabetic group } \\
& $\mathbf{r}$ & $\mathbf{p}$ & \multicolumn{1}{c}{$\mathbf{r}$} & $\mathbf{p}$ \\
\hline postoperative CMT & 0.271 & 0.329 & -0.479 & 0.071 \\
postoperative RNFL thickness & -0.278 & 0.317 & 0.091 & 0.747 \\
\hline
\end{tabular}

*r: correlation coefficient, BCVA: Best Corrected Visual Acuity, CMT: Central Macular Thickness, RNFL: Retinal Nerve fiber Layer

Table (3) Correlation of postoperative Retinal Nerve Fiber Layer thickness with Central Macular Thickness among studied groups.

\begin{tabular}{|c|c|c|c|c|}
\hline & \multicolumn{4}{|c|}{ Postoperative CMT } \\
\hline & \multicolumn{2}{|c|}{ Non diabetic group } & \multicolumn{2}{|c|}{ Diabetic group } \\
\hline & $\mathbf{r}$ & p & $\mathbf{r}$ & $\mathbf{p}$ \\
\hline Preoperative RNFL thickness & 0.418 & 0.121 & 0.362 & 0.185 \\
\hline
\end{tabular}

*r: correlation coefficient, CMT: Central Macular Thickness, RNFL: Retinal Nerve fiber Layer.

Table (4) Correlation of age with postoperative Best Corrected Visual Acuity, Central Macular Thickness and Retinal Nerve Fiber Layer thickness among studied groups.

\begin{tabular}{|c|c|c|c|c|}
\hline & \multicolumn{4}{|c|}{ Age } \\
\hline & \multicolumn{2}{|c|}{ Non diabetic group } & \multicolumn{2}{|c|}{ Diabetic group } \\
\hline & $\mathbf{r}$ & p & $\mathbf{r}$ & $\mathbf{p}$ \\
\hline Postoperative BCVA & 0.012 & 0.966 & -0.239 & 0.390 \\
\hline Postoperative CMT & 0.627 & 0.012 & -0.283 & 0.306 \\
\hline Postoperative RNFL thickness & 0.368 & 0.177 & -0.323 & 0.240 \\
\hline
\end{tabular}

*r: correlation coefficient, BCVA: Best Corrected Visual Acuity, CMT: Central Macular Thickness, RNFL: Retinal Nerve fiber Layer.

Table (5) Correlation of pre with postoperative Retinal Nerve Fiber Layer thickness among studied groups.

\begin{tabular}{|c|c|c|c|c|}
\hline & \multicolumn{4}{|c|}{ Preoperative RNFL thickness } \\
\hline & \multicolumn{2}{|c|}{ Non diabetic group } & \multicolumn{2}{|c|}{ Diabetic group } \\
\hline & $\mathbf{r}$ & p & $\mathbf{r}$ & p \\
\hline Postoperative RNFL thickness & 0.895 & $<0.001$ & 0.822 & $<0.001$ \\
\hline
\end{tabular}

*r: correlation coefficient, RNFL: Retinal Nerve fiber Layer.

No significant correlations were found between postoperative RNFL thickness with CMT among studied groups.

Postoperative CMT showed significant positive correlation with age in non-diabetic group. Otherwise, no significant correlations were found between age with postoperative BCVA, CMT and RNFL thickness among studied groups. Table 4

Preoperative RNFL thickness showed significantly positive correlation with postoperative RNFL thickness in non-diabetic and diabetic groups. Table 5

\section{Discussion}

We conducted this study to detect the change in CMT and peripapillary RNFL after uneventful phacoemulsification in diabetic patients without retinopathy comparing to non- diabetic patients.

The patients were selected with intermediate cataract density allowing fundus examination and OCT images' capturing. There were 2 groups in the study; the diabetic group included 15 eyes from patients of less than 5 years duration of DM without DR.

The non-diabetic group included 15 eyes of nondiabetic patients. They all underwent uneventful phacoemulsification.

The preoperative CMT was $223.73 \pm 32.83 \mu \mathrm{m}$ and $250.27 \pm 17.78 \mu \mathrm{m}$, in the non-diabetic group and diabetic group respectively. And 30 days postoperative CMT was $240.20 \pm 34.54 \mu \mathrm{m}$ and $282.20 \pm$ $26.20 \mu \mathrm{m}$, in the non-diabetic group and diabetic group respectively.

Other studies detected similar change in diabetic patients as Katsimpris et al in 2012 [10], which included 98 patients (49 diabetic patients with type 2 $\mathrm{DM}$ and 49 healthy controls undergoing phacoemulsification. Their recorded pre-operative CMT were $202 \pm 23 \mu \mathrm{m}$ and $205 \pm 18 \mu \mathrm{m}$ in diabetic group and non-diabetic group respectively ( $\mathrm{p}$ value $>$ 
0.1). and post-operative CMT after one month were 267 $\pm 25 \mu \mathrm{m}$ ( $251 \mu \mathrm{m}$ to $288 \mu \mathrm{m})$ and $229 \pm 21 \mu \mathrm{m}$ ( $218 \mu \mathrm{m}$ to $249 \mu \mathrm{m})$ in diabetic and non-diabetic group respectively ( $\mathrm{p}$ value $<0.05$ )

Giansanti et al in 2013 [11] also showed an increase in CMT in diabetics, they evaluate the central macular thickness (CMT) after uneventful phacoemulsification in selected groups of patients (with ERM-high myope-diabetic without retinopathyhealthy group).In diabetic group, CMT preoperative was $247 \pm 93 \mu \mathrm{m}$ and at Day 30 postoperative $270 \pm$ $100 \mu \mathrm{m}$ with change $23.26 \mu \mathrm{m}$ ( $\mathrm{p}$ value $=0.026$ ), in the healthy group preoperative CMT was $214 \pm 42 \mu \mathrm{m}$. The Postoperative CMT increased by 0.08 microns per day $(\mathrm{p}=0.012)$. It didn't show any significant difference compared to preoperative CMT till 360th day postoperative it reached $241 \pm 49 \mu \mathrm{m}$ ( $\mathrm{p}$ value was 0.018).

Tsilimbaris et al in 2012 [12] had similar results; they studied the alteration in mean foveal thickness (MFT) after uncomplicated cataract surgery in patients with diabetes, glaucoma, ERM and healthy people (no history of ocular or systemic disease). They also showed an increase in MFT in diabetics as compared to all other groups. In healthy group Pre-operative MFT was $202.8 \pm 25.79 \mu \mathrm{m}$ and 30 days Postoperative was $213.5 \pm 41.55 \mu \mathrm{m}(\mathrm{p}<0.05)$. And in diabetic group pre MFT was $206.6 \pm 21.47 \mu \mathrm{m} 30$ days postoperative was $241.89 \pm 76.2 \mu \mathrm{m}$. The group of diabetic patients presented the greatest difference in MFT value when compared with all other groups.

Kim et al in 2007 [2] assessed in his study the incidence or progression of ME after cataract surgery in diabetic patients using Stratus OCT3 and correlating this with degree of DR or other risk factors. Eyes with no DR developed minimal thickening of $18 \mu \mathrm{m}$ and 14 $\mu \mathrm{m}$ at 1 and 3 months, respectively. Eyes with moderate or severe nonproliferative DR or proliferative DR developed thickening of $145 \mu \mathrm{m}$ and $131 \mu \mathrm{m}$ at 1 and 3 months, respectively.

Biro et al in 2006 [13] conducted a study that enrolled 71 eyes to detect the change in foveal and perifoveal thickness measured by OCT after phacoemulsification and IOL implantation. The preoperative value was $234.17 \pm 2.6 \mu \mathrm{m}(\mathrm{n}=536)$ on the $6.0 \mathrm{~mm}$ area. After 1 week this value increased to $242.57 \pm 2.6 \mu \mathrm{m}(\mathrm{n}=488)$, after 1 month it increased to $247.77 \pm 4.6 \mathrm{~mm}(\mathrm{n}=352)$, and after 2 months it increased to $246.07 \pm 5.9 \mu \mathrm{m}(\mathrm{n}=208)$, which proved to be significant $(\mathrm{P}<0.05)$. The change of the $6.0 \mathrm{~mm}$ diameter perifoveal area after 1 month was $5.6 \%$. And this change is more or less similar to the change noted in the non-diabetic group of our study.

In 2009 Biro et al study, [14] the perifoveal retinal thickness changes in 18 eyes of DM patients compared to non-diabetic group which include 53 eyes. The mean duration of diabetes was $49.9 \pm 23.4$ months (mean \pm SD, 3-120 months). The preoperative value of macular thickness did not correlate to the duration of diabetic anamnesis. However In our study, duration of diabetes was $3.08 \pm 1.34$ years and it showed significant correlation between it and CMT change $(\mathrm{p}=0.004)$. In Biro et al. study, the foveal thickness in diabetic group was preoperatively $=204.1 \pm 6.2 \mu \mathrm{m}$ and $218.4 \pm 6.9 \mu \mathrm{m}$ after 1 month postoperative which proved to be significant $(\mathrm{p}=0.015)$

The foveal thickness in non-diabetic group was preoperatively $=198.0 \pm 4.3 \mu \mathrm{m}$ and $212.9 \pm 7.7 \mu \mathrm{m} 1$ month post-operative which proved to be significant ( $\mathrm{p}=0.047)$. The preoperative thickness of foveal region in the DM group was slightly higher with only $3 \%$ as compared to normal group. The thickness change percent in diabetic group: non-diabetic group was $7.4 \%$ : $5.6 \%$, comparing to our study it was $17.9 \%$ : $4.8 \%$.

Georgopoulos et al in 2008 [15] also study the central foveal thickness in a prospective study, 79 eyes were assessed by OCT, on day 1 , and weeks 2 and 4 after uncomplicated phacoemulsification. Pre-operative CMT was $150.4 \pm 18.84 \mu \mathrm{m}$ and postoperative week 4 was $152.00 \pm 17.11 \mu \mathrm{m}$. There wasn't statistically significant difference $(\mathrm{P}$ Value $=0.22)$. This change in CMT is less than that of our study.

In the non-diabetic group the pre-operative CMT was $211.6 \pm 19.6 \mu \mathrm{m}$ and post- operative $255.8 \pm 83.8$ $\mu \mathrm{m}$, the difference between the diabetic and nondiabetic group was statistically insignificant ( $\mathrm{P}$ value $>$ 0.05). However the difference between the preoperative and postoperative CMT in each group was statistically significant. The change of CMT was more than the change denoted in our study either in non-diabetic or diabetic group.

On the other hand, Degenring et al in 2007 [16], studied the uncomplicated phacoemulsification effect on CMT of diabetic and non-diabetic patients using Stratus OCT3. The study included 84 eyes from nondiabetic and 24 eyes from diabetic patients (18 did not show signs of DR in biomicroscopy, two had mild non proliferative DR, two had had panretinal laser photocoagulation, and two had had central and panretinal laser photocoagulation).Pre-operative MFT were $187 \pm 28 \mu \mathrm{m}$ and $182 \pm 27 \mu \mathrm{m}$ in diabetic and healthy group respectively (which did not differ between the diabetic and the non-diabetic groups $(\mathrm{p}=0.478))$.

\section{Conclusion}

In conclusion, DME is the most frequent sightthreatening complication of diabetic retinopathy, particularly in older type 2 diabetic patients. The possible onset of macular alterations after uncomplicated cataract surgery must be taken into account as a potential later complication, because it can lead to a permanent loss of visual acuity. With the help of the objective, noninvasive, good reproducible OCT measurements, the anatomical changes of the macula in the postoperative period can be well followed.

Macular thickness was found to be increased following cataract surgeries even after small-incision uneventful cataract surgery either in diabetic or in 
nondiabetic patients. The range of increased CMT is more in diabetic patients. Despite increased CMT, BCVA improved postoperatively in both groups. It is therefore important to distinguish which specific factors in individual patients who develop CME, so that targeted therapies may be developed. It is also equally important to diagnose CME without delay because early diagnosis means early treatment which in turns is associated with a better prognosis and for better visual outcome.

\section{References}

[1] M.L.Nelson and A.Martidis, "Managing cystoid macular edema after cataract surgery," Curr. Opin. Ophthalmol., vol. 14, pp. 39-43, 2003.

[2] S. J. Kim, R. Equi, and N. M. Bressler, "Analysis of macular edema after cataract surgery in patients with diabetes using optical coherence tomography," Ophthalmology, vol. 114, pp. 881-889, 2007.

[3] B.von Jagow, C.Ohrloff, and T. Kohnen, "Macular thickness after uneventful cataract surgery determined by optical coherence tomography," Graefe's Arch. Clin. Exp. Ophthalmol., vol. 245, pp. 1765-1771, 2007.

[4] El-Ashry M, Appaswamy S, Deokule S, Pagliarini S. The effect of phacoemulsification cataract surgery on the measurement of retinal nerve fiber layer thickness using optical coherence tomography. Curr-Eye-Res. 31(5): 409-13; May 2006.

[5] van Velthoven ME, van der Linden $\mathrm{MH}$, de Smet MD et al. Influence of cataract on optical coherence tomography image quality and retinal thickness. Br J Ophthalmol. 90:1159-1162; 2006.

[6] W.Goebel and T. Kretzchmar-Gross, "Retinal thickness in diabetic retinopathy: a study using optical coherence tomography (OCT)," Retina, vol. 22, pp. 759-767, 2002.

[7] A. M. Joussen et al., "A central role for inflammation in the pathogenesis of diabetic retinopathy," FASEB J., vol. 18, pp. 14501452, 2004.

[8] A. S. Shah and S. H. Chen, "Cataract surgery and diabetes," Curr. Opin. Ophthalmol., vol. 21, pp. 4-9, 2010.

[9] A.E.-K.I. El-Saadani, S.S.Mandour, and M. F. Laymouna, "Evaluation of macular changes after uncomplicated phacoemulsification surgery in diabetic patients using optical coherence tomography," Menoufia Med. J., vol. 31,pp. 289, 2018.

[10] J. M. Katsimpris, I. K. Petropoulos, G. Zoukas, T. Patokos, C. K. Brinkmann, and P. E. Theoulakis, "Central foveal thickness before and after cataract surgery in normal and in diabetic patients without retinopathy," Klin. Monbl. Augenheilkd., vol. 229, pp. 331-337, 2012.
[11] F. Giansanti et al., "Evaluation of macular thickness after uncomplicated cataract surgery using optical coherence tomography," Eur. J. Ophthalmol., vol. 23, no. 5, pp. 751-756, 2013.

[12] M. Tsilimbaris et al., "Prospective study of foveal thickness alterations after cataract surgery assessed by optical coherence tomography," Ophthalmologica, vol. 228, pp. 53-58, 2012.

[13] Z. Biro, Z. Balla, and B. Kovacs, "Change of foveal and perifoveal thickness measured by OCT after phacoemulsification and IOL implantation," Eye, vol. 22, pp. 8-12, 2008.

[14] Z. Biro and Z. Balla, "Foveal and perifoveal retinal thickness measured by OCT in diabetic patients after phacoemulsification cataract surgery.," Oftalmol. (Bucharest, Rom. 1990), vol. 53, pp. 54-60, 2009.

[15] G. T. Georgopoulos, D. Papaconstantinou, M. Niskopoulou, M. Moschos, I. Georgalas, and C. Koutsandrea, "Foveal thickness after phacoemulsification as measured by optical coherence tomography," Clin. Ophthalmol. (Auckland, NZ), vol. 2, pp. 817, 2008.

[16] R. F. Degenring, S. Vey, B. Kamppeter, W. M. Budde, J. B. Jonas, and G. Sauder, "Effect of uncomplicated phacoemulsification on the central retina in diabetic and non-diabetic subjects," Graefe's Arch. Clin. Exp. Ophthalmol., vol. 245, pp. 18-23, 2007. 